\title{
Uniqueness of Periodic Solution for a Class of Liénard $p$-Laplacian Equations
}

\author{
Fengjuan Cao, ${ }^{1}$ Zhenlai Han, $^{1,2}$ Ping Zhao, ${ }^{2}$ and Shurong Sun ${ }^{1,3}$ \\ ${ }^{1}$ School of Science, University of Jinan, Jinan, Shandong 250022, China \\ ${ }^{2}$ School of Control Science and Engineering, Shandong University, Jinan, Shandong 250061, China \\ ${ }^{3}$ Department of Mathematics and Statistics, Missouri University of Science and Technology, \\ Rolla, MO 65409-0020, USA \\ Correspondence should be addressed to Zhenlai Han, hanzhenlai@163.com
}

Received 31 December 2009; Accepted 23 February 2010

Academic Editor: Gaston Mandata N'Guerekata

Copyright (c) 2010 Fengjuan Cao et al. This is an open access article distributed under the Creative Commons Attribution License, which permits unrestricted use, distribution, and reproduction in any medium, provided the original work is properly cited.

\begin{abstract}
By topological degree theory and some analysis skills, we consider a class of generalized Liénard type $p$-Laplacian equations. Upon some suitable assumptions, the existence and uniqueness of periodic solutions for the generalized Liénard type $p$-Laplacian differential equations are obtained. It is significant that the nonlinear term contains two variables.
\end{abstract}

\section{Introduction}

As it is well known, the existence of periodic and almost periodic solutions is the most attracting topics in the qualitative theory of differential equations due to their vast applications in physics, mathematical biology, control theory, and others. More general equations and systems involving periodic boundary conditions have also been considered. Especially, the existence of periodic solutions for the Duffing equation, Rayleigh equation, and Liénard type equation, which are derived from many fields, such as fluid mechanics and nonlinear elastic mechanics, has received a lot of attention.

Many experts and scholars, such as Manásevich, Mawhin, Gaines, Cheung, Ren, $\mathrm{Ge}, \mathrm{Lu}$, and $\mathrm{Yu}$, have contributed a series of existence results to the periodicity theory of differential equations. Fixed point theory, Mawhin's continuation theorem, upper and lower solutions method, and coincidence degree theory are the common tools to study the periodicity theory of differential equations. Among these approaches, the Mawhin's continuation theorem seems to be a very powerful tool to deal with these problems.

Some contributions on periodic solutions to differential equations have been made in [1-13]. Recently, periodic problems involving the scalar $p$-Laplacian were studied by 
many authors. We mention the works by Manásevich and Mawhin [3] and Cheung and Ren $[4,8,10]$.

In [3], Manásevich and Mawhin investigated the existence of periodic solutions to the boundary value problem

$$
\left(\phi\left(u^{\prime}\right)\right)^{\prime}=f\left(t, u, u^{\prime}\right), \quad u(0)=u(T), \quad u^{\prime}(0)=u^{\prime}(T)
$$

where the function $\phi: R^{N} \rightarrow R^{N}$ is quite general and satisfies some monotonicity conditions which ensure that $\phi$ is homeomorphism onto $R^{N}$. Applying Leray-Schauder degree theory, the authors brought us the widely used Manásevich-Mawhin continuation theorem. When $\phi=\phi_{p}: R \rightarrow R$ is the so-called one-dimensional $p$-Laplacian operator given by $\phi_{p}(s)=$ $|s|^{p-2} s$.

Recently, by Mawhin's continuation theorem, Cheung and Ren studied the existence of $T$-periodic solutions for a $p$-Laplacian Liénard equation with a deviating argument in [4] as follows:

$$
\left(\varphi_{p}\left(x^{\prime}(t)\right)\right)^{\prime}+f(x(t)) x^{\prime}(t)+g(x(t-\tau(t)))=e(t)
$$

and two results (Theorems 3.1 and 3.2 ) on the existence of periodic solutions were obtained.

Ge and Ren [5] promoted Mawhin's continuation theorem to the case which involved the quasilinear operator successfully; this also prepared conditions for using Mawhin's continuation theorem to solve nonlinear boundary value problem.

Liu [7] has dealt with the existence and uniqueness of $T$-periodic solutions of the Liénard type $p$-Laplacian differential equation of the form

$$
\left(\varphi_{p}\left(x^{\prime}(t)\right)\right)^{\prime}+f(x(t)) x^{\prime}(t)+g(x(t))=e(t)
$$

by using topological degree theory, and one sufficient condition for the existence and uniqueness of $T$-periodic solutions of this equation was established.

The aim of this paper is to study the existence of periodic solutions to a class of $p$ Laplacian Liénard equations as follows:

$$
\left(\varphi_{p}\left(x^{\prime}(t)\right)\right)^{\prime}+f(t, x(t)) x^{\prime}(t)+g(t, x(t))=e(t),
$$

where $p>2, \varphi_{p}: R \rightarrow R$ is given by $\varphi_{p}(s)=|s|^{p-2} s$ for $s \neq 0, \varphi_{p}(0)=0, f \in C^{2}\left(R^{2}, R\right)$, $g \in C^{1}\left(R^{2}, R\right)$ and $T$-periodic in the first variable, where $T>0$ is a given constant, $e \in C(R, R)$, and $e(t+T)=e(t)$.

The paper is organized as follows. In Section 2, we give the definition of norm in Banach space and the main lemma. In Section 3, combining Lemma 2.1 with some analysis skills, two sufficient conditions about the existence of solutions for (1.4) are obtained. The nonlinear terms $f$ and $g$ contain two variables in this paper, which is seldom considered in the other papers, and the results are new. 


\section{Preliminary Results}

For convenience, we define

$$
C_{T}^{1}:=\left\{x \in C^{1}(R, R) ; x \text { is } T \text {-periodic }\right\}
$$

and the norm $\|\cdot\|$ is defined by $\|x\|=\max \left\{|x|_{\infty},\left|x^{\prime}\right|_{\infty}\right\}$, for all $x$,

$$
|x|_{\infty}=\max _{t \in[0, T]}|x(t)|, \quad\left|x^{\prime}\right|_{\infty}=\max _{t \in[0, T]}\left|x^{\prime}(t)\right|, \quad|x|_{k}=\left(\int_{0}^{T}|x(t)|^{k} d t\right)^{1 / k} .
$$

Clearly, $C_{T}^{1}$ is a Banach space endowed with such norm.

For the periodic boundary value problem

$$
\left(\varphi_{p}\left(x^{\prime}(t)\right)\right)^{\prime}=\tilde{f}\left(t, x, x^{\prime}\right), \quad x(0)=x(T), \quad x^{\prime}(0)=x^{\prime}(T),
$$

where $\tilde{f}$ is a continuous function and $T$-periodic in the first variable, we have the following result.

Lemma 2.1 (see [3]). Let $\Omega$ be an open bounded set in $C_{T}^{1}$. If the following conditions hold:

(i) for each $\lambda \in(0,1)$, the problem

$$
\left(\varphi_{p}\left(x^{\prime}(t)\right)\right)^{\prime}=\lambda \tilde{f}\left(t, x, x^{\prime}\right), \quad x(0)=x(T), \quad x^{\prime}(0)=x^{\prime}(T)
$$

has no solution on $\partial \Omega$,

(ii) the equation

$$
F(a):=\frac{1}{T} \int_{0}^{T} \tilde{f}(t, a, 0) d t=0
$$

has no solution on $\partial \Omega \cap R$,

(iii) the Brouwer degree of $F$ is $\operatorname{deg}\{F, \Omega \cap R, 0\} \neq 0$,

then the periodic boundary value problem (2.3) has at least one T-periodic solution on $\bar{\Omega}$.

Set

$$
\Psi(t, x(t))=\int_{0}^{x(t)} f(t, s) d s, \quad y(t)=\varphi\left(x^{\prime}(t)\right)+\Psi(t, x(t)) .
$$


We can rewrite (1.4) in the following form:

$$
\begin{gathered}
x^{\prime}(t)=|y(t)-\Psi(t, x(t))|^{q-1} \operatorname{sgn}(y(t)-\Psi(t, x(t))), \\
y^{\prime}(t)=\int_{0}^{x(t)} \frac{\partial}{\partial t} f(t, s) d s-g(t, x(t))+e(t),
\end{gathered}
$$

where $1<q<2$ and $1 / p+1 / q=1$.

Lemma 2.2. Suppose the following condition holds:

$\left(A_{1}\right) \partial f / \partial t-\partial g /\left.\partial s\right|_{(t, s)}>0$, for all $t \in R$.

Then (1.4) has at most one T-periodic solution.

Proof. Let $x_{1}(t)$ and $x_{2}(t)$ be two T-periodic solutions of (1.4). Then, from (2.7), we obtain

$$
\begin{aligned}
& x_{i}^{\prime}(t)=\left|y_{i}(t)-\Psi\left(t, x_{i}(t)\right)\right|^{q-1} \operatorname{sgn}\left(y_{i}(t)-\Psi\left(t, x_{i}(t)\right)\right) \\
& y_{i}^{\prime}(t)=\int_{0}^{x_{i}(t)} \frac{\partial}{\partial t} f(t, s) d s-g\left(t, x_{i}(t)\right)+e(t)
\end{aligned}
$$

Set

$$
v(t)=x_{1}(t)-x_{2}(t), \quad u(t)=y_{1}(t)-y_{2}(t)
$$

Then it follows from (2.8) that

$$
\begin{aligned}
v^{\prime}(t)= & \left|y_{1}(t)-\Psi\left(t, x_{1}(t)\right)\right|^{q-1} \operatorname{sgn}\left(y_{1}(t)-\Psi\left(t, x_{1}(t)\right)\right) \\
& -\left|y_{2}(t)-\Psi\left(t, x_{2}(t)\right)\right|^{q-1} \operatorname{sgn}\left(y_{2}(t)-\Psi\left(t, x_{2}(t)\right)\right), \\
u^{\prime}(t)= & \int_{x_{2}(t)}^{x_{1}(t)}\left(\frac{\partial f}{\partial t}-\frac{\partial g}{\partial s}\right)(t, s) d s .
\end{aligned}
$$

We claim that $u(t) \leq 0$ for all $t \in R$. By way of contradiction, in view of $u \in C^{2}[0, T]$ and $u(t+T)=u(t)$ for all $t \in R$, we obtain $\max _{t \in R} u(t)>0$. Then there must exist $t^{*} \in R$; for convenience, we can choose $t^{*} \in[0, T]$ such that

$$
u\left(t^{*}\right)=\max _{t \in[0, T]} u(t)=\max _{t \in R} u(t)>0,
$$


which implies that

$$
\begin{aligned}
u^{\prime}\left(t^{*}\right)= & \left.\int_{x_{1}(t)}^{x_{2}(t)}\left(\frac{\partial f}{\partial t}-\frac{\partial g}{\partial s}\right)(t, s) d s\right|_{t=t^{*}}=0, \\
u^{\prime \prime}\left(t^{*}\right)= & \left.\left(\int_{x_{2}(t)}^{x_{1}(t)} \frac{\partial}{\partial t} f(t, s) d s\right)^{\prime}\right|_{t=t^{*}} \\
& -\left.\left[\frac{\partial}{\partial t} g\left(t, x_{1}(t)\right)+\frac{\partial}{\partial x_{1}} g\left(t, x_{1}(t)\right) x_{1}^{\prime}(t)-\frac{\partial}{\partial t} g\left(t, x_{2}(t)\right)-\frac{\partial}{\partial x_{2}} g\left(t, x_{2}(t)\right) x_{2}^{\prime}(t)\right]\right|_{t=t^{*}} \leq 0 .
\end{aligned}
$$

Set

$$
\Delta_{1}=\left\{t: t \in[0, T], x_{1}(t) \neq x_{2}(t)\right\}, \quad \Delta_{2}=\left\{t: t \in[0, T], x_{1}(t)=x_{2}(t)\right\} .
$$

Then, $[0, T]=\Delta_{1} \cup \Delta_{2}$. Since $t^{*} \in[0, T]$, if $t^{*} \in \Delta_{1}$, from the first equation of (2.12), we have

$$
\left.\left(\int_{x_{1}(t)}^{x_{2}(t)}\left(\frac{\partial f}{\partial t}-\frac{\partial g}{\partial s}\right)(t, s) d s\right)\right|_{t=t^{*}}=0
$$

which contradicts assumption $\left(A_{1}\right)$, so $t^{*} \bar{\epsilon} \Delta_{1}$; it implies that $t^{*} \in \Delta_{2}$, that is, $x_{1}\left(t^{*}\right)=x_{2}\left(t^{*}\right)$. Hence we have

$$
\begin{gathered}
\left.\frac{\partial}{\partial t} f\left(t, x_{1}(t)\right)\right|_{t=t^{*}}=\left.\frac{\partial}{\partial t} f\left(t, x_{2}(t)\right)\right|_{t=t^{*}},\left.\quad \frac{\partial}{\partial x_{1}} g\left(t, x_{1}(t)\right)\right|_{t=t^{*}}=\left.\frac{\partial}{\partial x_{2}} g\left(t, x_{2}(t)\right)\right|_{t=t^{*}}, \\
\left.\left(\int_{x_{1}(t)}^{x_{2}(t)} \frac{\partial}{\partial t} f(t, s) d s\right)^{\prime}\right|_{t=t^{*}}=\left.\frac{\partial}{\partial t} f\left(t, x_{1}(t)\right)\right|_{t=t^{*}}\left[x_{1}^{\prime}\left(t^{*}\right)-x_{2}^{\prime}\left(t^{*}\right)\right] .
\end{gathered}
$$

Substituting (2.15) into the second equation of (2.12), we have

$$
\begin{aligned}
u^{\prime \prime}\left(t^{*}\right)= & \left.\frac{\partial}{\partial t} f\left(t, x_{1}(t)\right)\right|_{t=t^{*}}\left[x_{1}^{\prime}\left(t^{*}\right)-x_{2}^{\prime}\left(t^{*}\right)\right]-\left.\frac{\partial}{\partial x_{1}} g\left(t, x_{1}(t)\right)\right|_{t=t^{*}}\left[x_{1}^{\prime}\left(t^{*}\right)-x_{2}^{\prime}\left(t^{*}\right)\right] \\
= & {\left.\left[\frac{\partial}{\partial t} f\left(t, x_{1}(t)\right)-\frac{\partial}{\partial x_{1}} g\left(t, x_{1}(t)\right)\right]\right|_{t=t^{*}}\left[x_{1}^{\prime}\left(t^{*}\right)-x_{2}^{\prime}\left(t^{*}\right)\right] } \\
= & {\left.\left[\frac{\partial f}{\partial t}-\frac{\partial g}{\partial x_{1}}\right]\right|_{\left(t^{*}, x_{1}\left(t^{*}\right)\right)} } \\
& \times\left[\left|y_{1}\left(t^{*}\right)-\Psi\left(t^{*}, x_{1}\left(t^{*}\right)\right)\right|^{q-1} \operatorname{sgn}\left(y_{1}\left(t^{*}\right)-\Psi\left(t^{*}, x_{1}\left(t^{*}\right)\right)\right)\right. \\
& \left.\quad-\left|y_{2}\left(t^{*}\right)-\Psi\left(t^{*}, x_{2}\left(t^{*}\right)\right)\right|^{q-1} \operatorname{sgn}\left(y_{2}\left(t^{*}\right)-\Psi\left(t^{*}, x_{2}\left(t^{*}\right)\right)\right)\right] .
\end{aligned}
$$


Noticing $\left(A_{1}\right)$ and that $\left.\left[\partial f / \partial t-\partial g / \partial x_{1}\right]\right|_{\left(t^{*}, x_{1}\left(t^{*}\right)\right)}>0$ and $u\left(t^{*}\right)=y_{1}\left(t^{*}\right)-y_{2}\left(t^{*}\right)$, from (2.16), we know that

$$
u^{\prime \prime}\left(t^{*}\right)>0,
$$

this contradicts the second equation of (2.12). So we have $u(t)=y_{1}(t)-y_{2}(t) \leq 0$, for all $t \in R$.

By using a similar argument, we can also show that

$$
y_{2}(t)-y_{1}(t) \leq 0, \quad \forall t \in R
$$

Then, from (2.10) we obtain

$$
\int_{x_{1}(t)}^{x_{2}(t)}\left(\frac{\partial f}{\partial t}-\frac{\partial g}{\partial x}\right)(t, s) d s \equiv 0
$$

For every $t \in[0, T]$, if $t \in \Delta_{1}$, then it contradicts (2.19), so $\Delta_{1}=\emptyset$; it implies that $[0, T]=\Delta_{2}$, then $x_{1}(t) \equiv x_{2}(t)$, for all $t \in[0, T]$.

Hence, (1.4) has at most one $T$-periodic solution. The proof of Lemma 2.2 is completed now.

\section{Main Results}

Theorem 3.1. Let $\left(A_{1}\right)$ hold. Suppose that there exists a positive constant $d$ such that

$$
\begin{aligned}
& \left(A_{2}\right) x(g(t, x)-e(t))<0, \text { for all }|x|>d, \\
& \left(A_{3}\right) \lim _{u \rightarrow+\infty}\left|f(t, u) / u^{p-1}\right|=r>0 .
\end{aligned}
$$

Then (1.4) has one unique T-periodic solution if $\left(1 / 2^{p-1}\right)(r+\varepsilon) \lambda T^{p-1}<1$.

Proof. Consider the homotopic equation of (1.4) as follows:

$$
\left(\varphi_{p}\left(x^{\prime}(t)\right)\right)^{\prime}+\lambda f(t, x(t)) x^{\prime}(t)+\lambda g(t, x(t))=\lambda e(t), \quad \lambda \in(0,1) .
$$

By Lemma 2.2, combining $\left(A_{1}\right)$, it is easy to see that (1.4) has at least one $T$-periodic solution. For the remainder, we will apply Lemma 2.1 to study (3.1). Firstly, we will verify that all the possible $T$-periodic solutions of (3.1) are bounded.

Let $x \in C_{T}^{1}$ be an arbitrary solution of (3.1) with period $T$. By integrating the two sides of (3.1) from 0 to $T$ and $x^{\prime}(0)=x^{\prime}(T)$, we obtain

$$
\int_{0}^{T} f(t, x(t)) x^{\prime}(t) d t+\int_{0}^{T}[g(t, x(t))-e(t)] d t=0
$$


Consider $x(0)=x(T)$ and $x \in C_{T}^{1}$, there exists $t_{0} \in[0, T]$ such that $x^{\prime}\left(t_{0}\right)=0$, while for $\varphi_{p}(0)=0$ we see that

$$
\left|\varphi_{p}\left(x^{\prime}(t)\right)\right|=\left|\int_{t_{0}}^{t}\left(\varphi_{p}\left(x^{\prime}(t)\right)\right)^{\prime} d s\right| \leq \lambda \int_{0}^{T}|f(t, x(t))|\left|x^{\prime}(t)\right| d t+\lambda \int_{0}^{T}|g(t, x(t)) e(t)| d t,
$$

where $t \in\left[t_{0}, t_{0}+T\right]$. Let $\bar{t}$ be the global maximum point of $x(t)$ on $[0, T]$. Then as $x^{\prime}(\bar{t})=0$, we claim that

$$
\left(\varphi_{p}\left(x^{\prime}(\bar{t})\right)\right)^{\prime}=\left(\left|x^{\prime}(\bar{t})\right|^{p-2} x^{\prime}(\bar{t})\right)^{\prime} \leq 0
$$

Otherwise, we have $\left(\varphi_{p}\left(x^{\prime}(\bar{t})\right)\right)^{\prime}>0$, there must exist a constant $\varepsilon>0$ such that $\left(\varphi_{p}\left(x^{\prime}(t)\right)\right)^{\prime}=$ $\left(\left|x^{\prime}(t)\right|^{p-2} x^{\prime}(t)\right)^{\prime}>0$, for $t \in(\bar{t}-\varepsilon, \bar{t}+\varepsilon)$; therefore, $\varphi_{p}\left(x^{\prime}(t)\right)$ is strictly increasing for $t \in(\bar{t}-\varepsilon, \bar{t}+\varepsilon)$, which implies that $x^{\prime}(t)$ is strictly increasing for $t \in(\bar{t}-\varepsilon, \bar{t}+\varepsilon)$. Thus, (3.4) is true. Then

$$
g(\bar{t}, x(\bar{t}))-e(\bar{t}) \geq 0
$$

In view of $\left(A_{2}\right),(3.5)$ implies that $x(\bar{t}) \leq d$; similar to the global minimum point of $x(t)$ on $[0, T]$. Since $x(t) \in C_{T}^{1}$, it follows that there exists a constant $\xi \in[0, T]$ such that $|x(\xi)| \leq d$. Then we have

$$
\begin{gathered}
|x(t)|=\left|x(\xi)+\int_{\xi}^{t} x^{\prime}(s) d s\right| \leq d+\int_{\xi}^{t}\left|x^{\prime}(s)\right| d s, \quad t \in[\xi, \xi+T], \\
|x(t)|=|x(t-T)|=\left|x(\xi)-\int_{t-T}^{\xi} x^{\prime}(s) d s\right| \leq d+\int_{t-T}^{\xi}\left|x^{\prime}(s)\right| d s, \quad t \in[\xi, \xi+T] .
\end{gathered}
$$

Combining the above two inequalities, we obtain

$$
\begin{aligned}
|x|_{\infty} & =\max _{t \in[0, T]}|x(t)|=\max _{t \in[\xi, \xi+T]}|x(t)| \\
& \leq \max _{t \in[\xi, \xi+T]}\left\{d+\frac{1}{2}\left(\int_{\xi}^{t}\left|x^{\prime}(s)\right| d s+\int_{t-T}^{\xi}\left|x^{\prime}(s)\right| d s\right)\right\} \\
& \leq d+\frac{1}{2} \int_{0}^{T}\left|x^{\prime}(s)\right| d s .
\end{aligned}
$$

Considering $\left(A_{3}\right)$, there exist constants $d_{1}$ and the sufficiently small $\varepsilon>0$ such that

$$
|f(t, x(t))| \leq(r+\varepsilon)|x(t)|^{p-1}, \quad \text { when }|x(t)|>d_{1} .
$$


Set

$$
\begin{aligned}
\Delta_{1}=\left\{t: t \in[0, T],|x(t)|>d_{1}\right\}, & \Delta_{2}=\left\{t: t \in[0, T],|x(t)| \leq d_{1}\right\}, \\
E_{1}=\{t: t \in[0, T],|x(t)|>d\}, & E_{2}=\{t: t \in[0, T],|x(t)| \leq d\} .
\end{aligned}
$$

From (3.7), we have

$$
\begin{aligned}
\int_{0}^{T} f(t, x(t)) x^{\prime}(t) x(t) d t & =\int_{\Delta_{1}} f(t, x(\mathrm{t})) x^{\prime}(t) x(t) d t+\int_{\Delta_{2}} f(t, x(t)) x^{\prime}(t) x(t) d t \\
& \leq f_{A}|x(t)|_{\infty} \int_{0}^{T}\left|x^{\prime}(t)\right| d t+(r+\varepsilon)|x(t)|_{\infty}^{p-1} \int_{0}^{T}\left|x^{\prime}(t)\right| d t \\
& \leq(r+\varepsilon)\left(d+\frac{1}{2} \int_{0}^{T}\left|x^{\prime}(t)\right| d t\right)^{p-1} \int_{0}^{T}\left|x^{\prime}(t)\right| d t+\frac{1}{2} f_{A}\left(\int_{0}^{T}\left|x^{\prime}(t)\right| d t\right)^{2} \\
& +d f_{A} \int_{0}^{T}\left|x^{\prime}(t)\right| d t
\end{aligned}
$$

where $f_{A}=\max \left\{|f(t, x(t))|: t \in \Delta_{2}\right\}$. Combining the classical inequality $(1+x)^{p} \leq 1+(p+1) x$, when $x \in[0, h(p))$, where $h(p)$ is a constant, since

$$
\left(d+\frac{1}{2} \int_{0}^{T}\left|x^{\prime}(t)\right| d t\right)^{p-1}=\left(\frac{1}{2} \int_{0}^{T}\left|x^{\prime}(t)\right| d t\right)^{p-1}\left(1+\frac{2 d}{\int_{0}^{T}\left|x^{\prime}(t)\right| d t}\right)^{p-1}
$$

then we consider the following two cases.

Case 1. If $2 d / \int_{0}^{T}\left|x^{\prime}(t)\right| d t \geq h(p)$, then $\int_{0}^{T}\left|x^{\prime}(t)\right| d t \leq 2 d / h(p)$. Combining (3.7), we know that

$$
\begin{gathered}
|x|_{\infty} \leq d+\frac{1}{2} \int_{0}^{T}\left|x^{\prime}(s)\right| d s \leq d+\frac{2 d}{h(p)} \\
\left(d+\frac{1}{2} \int_{0}^{T}\left|x^{\prime}(t)\right| d t\right)^{p}=\left(\frac{1}{2} \int_{0}^{T}\left|x^{\prime}(t)\right| d t\right)^{p}\left(1+\frac{2 d}{\int_{0}^{T}\left|x^{\prime}(t)\right| d t}\right)^{p},
\end{gathered}
$$

when $2 d / \int_{0}^{T}\left|x^{\prime}(t)\right| d t \geq h(p)$, then we have $\int_{0}^{T}\left|x^{\prime}(t)\right| d t \leq 2 d / h(p)$.

Case 2. When $0<2 d / \int_{0}^{T}\left|x^{\prime}(t)\right| d t<h(p)$, then from the above classical inequality, we obtain

$$
\left(d+\frac{1}{2} \int_{0}^{T}\left|x^{\prime}(t)\right| d t\right)^{p-1}=\left(\frac{1}{2} \int_{0}^{T}\left|x^{\prime}(t)\right| d t\right)^{p-1}\left(1+\frac{2 d p}{\int_{0}^{T}\left|x^{\prime}(t)\right| d t}\right) .
$$


Advances in Difference Equations

9

Substituting the above inequality into (3.10), we get

$$
\begin{aligned}
\int_{0}^{T} f(t, x(t)) x^{\prime}(t) x(t) d t \leq & \frac{1}{2^{p-1}}(r+\varepsilon)\left(\int_{0}^{T}\left|x^{\prime}(t)\right| d t\right)^{p}+\frac{1}{2^{p-2}} d p(r+\varepsilon)\left(\int_{0}^{T}\left|x^{\prime}(t)\right| d t\right)^{p-1} \\
& +\frac{1}{2} f_{A}\left(\int_{0}^{T}\left|x^{\prime}(t)\right| d t\right)^{2}+d f_{A} \int_{0}^{T}\left|x^{\prime}(t)\right| d t .
\end{aligned}
$$

Since $x(t)$ is $T$-periodic, multiplying $x(t)$ by (3.1) and then integrating from 0 to $T$, in view of $\left(A_{2}\right)$, we have

$$
\begin{aligned}
\int_{0}^{T}\left|x^{\prime}(t)\right|^{p} d t & =-\int_{0}^{T}\left|\varphi_{p}\left(x^{\prime}(t)\right)\right|^{\prime} x(t) d t \\
& =\lambda \int_{0}^{T} f(t, x(t)) x^{\prime}(t) x(t) d t+\lambda \int_{0}^{T} g(t, x(t)) x(t) d t-\lambda \int_{0}^{T} e(t) x(t) d t \\
& =\lambda \int_{0}^{T} f(t, x(t)) x^{\prime}(t) x(t) d t+\lambda \int_{0}^{T}[g(t, x(t))-e(t)] x(t) d t .
\end{aligned}
$$

Substituting (3.14) into (3.15) and since

$$
\left(\int_{0}^{T}|x(t)|^{r} d t\right)^{1 / r} \leq T^{(s-r) / s}\left(\left.\int_{0}^{T} x(t)\right|^{s} d t\right)^{1 / s}, \quad 0<r \leq s,
$$

we obtain

$$
\begin{aligned}
\int_{0}^{T}\left|x^{\prime}(t)\right|^{p} d t= & -\int_{0}^{T}\left|\varphi_{p}\left(x^{\prime}(t)\right)\right|^{\prime} x(t) d t \\
\leq & \frac{r+\varepsilon}{2^{p-1}} \lambda T^{p-1} \int_{0}^{T}\left|x^{\prime}(t)\right|^{p} d t+\frac{1}{2^{p-2}} \lambda T^{(p-1)^{2} / p} d p(r+\varepsilon)\left(\int_{0}^{T}\left|x^{\prime}(t)\right|^{p} d t\right)^{(p-1) / p} \\
& +\frac{1}{2} \lambda T^{2(p-1) / p} f_{A}\left(\int_{0}^{T}\left|x^{\prime}(t)\right|^{p} d t\right)^{2 / p}+\lambda T^{(p-1) / p} d f_{A}\left(\int_{0}^{T}\left|x^{\prime}(t)\right|^{p} d t\right)^{1 / p} \\
& +\lambda\left(d+\frac{1}{2} \int_{0}^{T}\left|x^{\prime}(t)\right| d t\right) \int_{0}^{T} \max \left\{|g(t, x(t))-e(t)|, t \in E_{2}\right\} d t \\
\leq & Q_{1} \int_{0}^{T}\left|x^{\prime}(t)\right|^{p} d t+Q_{2}\left(\int_{0}^{T}\left|x^{\prime}(t)\right|^{p} d t\right)^{(p-1) / p}+Q_{3}\left(\int_{0}^{T}\left|x^{\prime}(t)\right|^{p} d t\right)^{2 / p} \\
& +Q_{4}\left(\int_{0}^{T}\left|x^{\prime}(t)\right|^{p} d t\right)^{1 / p}+Q_{5},
\end{aligned}
$$


where

$$
\begin{gathered}
Q_{1}=\frac{1}{2^{p-1}}(r+\varepsilon) \lambda T^{p-1}, \quad Q_{2}=\frac{1}{2^{p-2}} \lambda d p(r+\varepsilon) T^{(p-1)^{2} / p}, \quad Q_{3}=\frac{1}{2} \lambda f_{A} T^{2(p-1) / p}, \\
Q_{4}=\lambda d f_{A} T^{(p-1) / p}+\frac{1}{2} \lambda T^{(p-1) / p} \int_{0}^{T} \max \left\{|g(t, x(t))-e(t)|, t \in E_{2}\right\} d t, \\
Q_{5}=\lambda d \int_{0}^{T} \max \left\{|g(t, x(t))-e(t)|, t \in E_{2}\right\} d t .
\end{gathered}
$$

Since $p>2$ and $\left(1 / 2^{p-1}\right)(r+\varepsilon) \lambda T^{p-1}<1$, from (3.17), we know that there exists a constant $M_{1}$ such that $\int_{0}^{T}\left|x^{\prime}(t)\right|^{p} d t \leq M_{1}$. Then,

$$
\left(\int_{0}^{T}\left|x^{\prime}(t)\right| d t\right)^{p} \leq T^{p-1} \int_{0}^{T}\left|x^{\prime}(t)\right|^{p} d t \leq T^{p-1} M_{1}:=N_{1} .
$$

So, there exists a constant such that

$$
|x(t)| \leq M_{2}, \quad\left|x^{\prime}(t)\right| \leq M_{2} .
$$

Set

$$
\Omega=\left\{x \in C_{T}^{1}:|x|_{\infty} \leq M_{2}+1,\left|x^{\prime}\right|_{\infty} \leq M_{2}+1\right\} .
$$

Then (3.1) has no solution on $\partial \Omega$ as $\lambda \in(0,1)$, and when $x(t) \in \partial \Omega \cap R, x(t)=M_{2}+1$ or $x(t)=-M_{2}-1$; from $\left(A_{2}\right)$, we can see that

$$
\begin{gathered}
\frac{1}{T} \int_{0}^{T}\left\{-g\left(t, M_{2}+1\right)+e(t)\right\} d t=-\frac{1}{T} \int_{0}^{T}\left\{g\left(t, M_{2}+1\right)-e(t)\right\} d t>0, \\
\frac{1}{T} \int_{0}^{T}\left\{-g\left(t,-M_{2}-1\right)+e(t)\right\} d t=-\frac{1}{T} \int_{0}^{T}\left\{g\left(t,-M_{2}-1\right)-e(t)\right\} d t<0,
\end{gathered}
$$

so condition (ii) holds.

Set

$$
H(x, \mu)=\mu x+(1-\mu) \frac{1}{T} \int_{0}^{T}\{-g(t, x)+e(t)\} d t .
$$

Then, when $x \in \partial \Omega \cap R, \mu \in[0,1]$, we have

$$
x H(x, \mu)=\mu x^{2}-(1-\mu) x \frac{1}{T} \int_{0}^{T}\{g(t, x)-e(t)\} d t>0,
$$


thus $H(x, \mu)$ is a homotopic transformation and

$$
\begin{aligned}
\operatorname{deg}\{F, \Omega \cap R, 0\} & =\operatorname{deg}\left\{-\frac{1}{T} \int_{0}^{T}\{g(t, x)-e(t)\} d t, \Omega \cap R, 0\right\} \\
& =\operatorname{deg}\{x, \Omega \cap R, 0\} \neq 0 .
\end{aligned}
$$

So condition (iii) holds. In view of Lemma 2.1, there exists at least one solution with period $T$. This completes the proof.

Theorem 3.2. Let $\left(A_{1}\right)$ hold. Suppose that there exist positive constants $d_{1}$ and $d_{2}$ satisfying the following conditions:

$$
\begin{aligned}
& \left(A_{4}\right)-\operatorname{sgn}(x) g(t, x)>|e|_{\infty}, \text { when }|x|>d_{1} ; \\
& \left(A_{5}\right) f(t, x(t))>0, t \in R ; \\
& \left(A_{6}\right)|g(t, x) / x| \leq l \text {, when }|x|>d_{1} .
\end{aligned}
$$

Then for (1.4) there exists one unique T-periodic solution when $\sigma>l T$.

Proof. We can rewrite (3.1) in the following from:

$$
\begin{gathered}
x_{1}^{\prime}(t)=\varphi_{q}\left(x_{2}(t)\right), \\
x_{2}^{\prime}(t)=-\lambda f\left(t, x_{1}(t)\right) x_{1}^{\prime}(t)-\lambda g\left(t, x_{1}(t)\right)+\lambda e(t) .
\end{gathered}
$$

Let $\left(x_{1}(t), x_{2}(t)\right) \in C_{T}^{1}$ be a $T$-periodic solution of (3.26), then $x_{1}(t)$ must be a $T$-periodic solution of (3.1). First we claim that there is a constant $\xi \in R$ such that

$$
\left|x_{1}(\xi)\right| \leq d
$$

Take $t_{0}, t_{1}$ as the global maximum point and global minimum point of $x(t)$ on $[0, T]$, respectively, then

$$
x_{1}^{\prime}\left(t_{0}\right)=x_{1}^{\prime}\left(t_{1}\right)=0, \quad x_{1}\left(t_{0}\right)=\max _{t \in R} x_{1}(t)=\max _{t \in[0, T]} x_{1}(t) .
$$

From the first equation of (3.26) we have $x_{2}(t)=\varphi_{p}\left(x_{1}^{\prime}(t)\right)$, so $x_{2}\left(t_{0}\right)=\varphi_{p}\left(x_{1}^{\prime}\left(t_{0}\right)\right)=0=x_{2}\left(t_{1}\right)$. We claim that

$$
x_{2}^{\prime}\left(t_{0}\right) \leq 0
$$

By way of contradiction, (3.29) does not hold, then $x_{2}^{\prime}\left(t_{0}\right)>0$. So there exists $\varepsilon>0$ such that $x_{2}^{\prime}(t)>0$, for $t \in\left(t_{0}-\varepsilon, t_{0}+\varepsilon\right)$; therefore, $x_{2}(t)>x_{2}\left(t_{0}\right)=0$, for $t \in\left[t_{0}, t_{0}+\varepsilon\right)$, so $x_{1}^{\prime}(t)=\varphi_{q}\left(x_{2}(t)\right)>0, t \in\left[t_{0}, t_{0}+\varepsilon\right)$, that is, $x_{1}(t)>x_{1}\left(t_{0}\right)$, for $t \in\left[t_{0}, t_{0}+\varepsilon\right)$. This contradicts the definition of $t_{0}$, so we have $x_{2}^{\prime}\left(t_{0}\right) \leq 0$. 
Substituting $t_{0}$ into the second equation of (3.26), we obtain

$$
g\left(t_{0}, x_{1}\left(t_{0}\right)\right)-e\left(t_{0}\right) \geq 0
$$

By condition $\left(A_{4}\right)$, we have $x_{1}\left(t_{0}\right) \leq d$. Similarly, we get $x_{1}\left(t_{1}\right) \geq-d$.

Case 1. If $x_{1}\left(t_{1}\right) \leq d$, define $\xi=t_{1}$. Obviously $\left|x_{1}(\xi)\right| \leq d$.

Case 2. If $x_{1}\left(t_{1}\right) \geq d$, from the fact that $x_{1}$ is a continuous function in $R$, there exists a constant $\xi$ between $t_{0}$ and $t_{1}$ such that $\left|x_{1}(\xi)\right|=d$.

So we have that (3.27) holds. Next, in view of $\xi \in R$, there are integer $k$ and constant $t^{*} \in[0, T]$ such that $\xi=k T+t^{*}$, hence $\left|x_{1}(\xi)\right|=\left|x_{1}\left(t^{*}\right)\right| \leq d$.

So

$$
\left|x_{1}(t)\right| \leq d+\int_{0}^{T}+\left|x^{\prime}(s)\right| d s
$$

We claim that all the periodic solutions $x$ of (3.1) are bounded and $|x(t)| \leq d+\int_{0}^{T}\left|x^{\prime}(s)\right| d s$.

Let

$$
E_{1}=\left\{t: t \in[0, T],|x(t)|>d_{1}\right\}, \quad E_{2}=\left\{t: t \in[0, T],|x(t)| \leq d_{1}\right\} .
$$

Multiplying both sides of (3.1) by $x^{\prime}(t)$ and integrating from 0 to $T$, together with $\left(A_{5}\right)$ and $\left(A_{6}\right)$, we have

$$
\begin{aligned}
\sigma \int_{0}^{T}\left|x^{\prime}(t)\right|^{2} d t & \leq \int_{0}^{T} f(t, x(t))\left|x^{\prime}(t)\right|^{2} d t \\
& \leq \int_{0}^{T}|g(t, x(t))|\left|x^{\prime}(t)\right| d t+\int_{0}^{T}|e(t)|\left|x^{\prime}(t)\right| d t \\
& \leq \int_{E_{1}}|g(t, x(t))|\left|x^{\prime}(t)\right| d t+\int_{E_{2}}|g(t, x(t))|\left|x^{\prime}(t)\right| d t+\int_{0}^{T}|e(t)|\left|x^{\prime}(t)\right| d t \\
& \leq l \int_{0}^{T}|x(t)|\left|x^{\prime}(t)\right| d t+|e|_{\infty} \int_{0}^{T}\left|x^{\prime}(t)\right| d t+g_{A} \int_{0}^{T}\left|x^{\prime}(t)\right| d t \\
& \leq l\left(d+\int_{0}^{T}\left|x^{\prime}(t)\right| d t\right) \int_{0}^{T}\left|x^{\prime}(t)\right| d t+\left(g_{A}+|e|_{\infty}\right) \int_{0}^{T}\left|x^{\prime}(t)\right| d t \\
& =\left(l d+g_{A}+|e|_{\infty}\right) \int_{0}^{T}\left|x^{\prime}(t)\right| d t+l T \int_{0}^{T}\left|x^{\prime}(t)\right|^{2} d t
\end{aligned}
$$


where $\sigma=\min \{f(t, x(t))\}$, and $g_{A}=\max \left\{|g(t, x(t))|:|x(t)| \leq d_{1}\right\}$. That is,

$$
(\sigma-l T) \int_{0}^{T}\left|x^{\prime}(t)\right|^{2} d t \leq\left(l d+g_{A}+|e|_{\infty}\right) \int_{0}^{T}\left|x^{\prime}(t)\right| d t
$$

Using Hölder's inequality and $\sigma>l T$, we have

$$
\frac{1}{T}\left(\int_{0}^{T}\left|x^{\prime}(t)\right| d t\right)^{2} \leq \int_{0}^{T}\left|x^{\prime}(t)\right|^{2} d t \leq \frac{l d+|e|_{\infty}+g_{A}}{\sigma-l T} \int_{0}^{T}\left|x^{\prime}(t)\right| d t,
$$

so

$$
\int_{0}^{T}\left|x^{\prime}(t)\right| d t \leq T \frac{l d+|e|_{0}+g_{A}}{\sigma-l T}:=M
$$

there must be a positive constant $M_{1}>M$ such that

$$
\int_{0}^{T}\left|x^{\prime}(t)\right| d t \leq M_{1}, \quad \int_{0}^{T}|x(t)| d t \leq M_{1}
$$

hence together with (3.31), we have $\left|x_{1}\right|_{\infty} \leq d+M_{1}:=M_{2}$.

This proves the claim and that the rest of the proof of the theorem is identical to that of Theorem 3.1.

\section{Acknowledgments}

The authors sincerely thank the reviewers for their valuable suggestions and useful comments that have led to the present improved version of the original manuscript. This research is supported by the Natural Science Foundation of China (60774004, 60904024), China Postdoctoral Science Foundation Funded Project (20080441126, 200902564), Shandong Postdoctoral Funded Project (200802018), and the Natural Scientific Foundation of Shandong Province (Y2008A28, ZR2009AL003) and is also supported by University of Jinan Research Funds for Doctors (XBS0843).

\section{References}

[1] S. Lu, "Existence of periodic solutions to a $p$-Laplacian Liénard differential equation with a deviating argument," Nonlinear Analysis: Theory, Methods E Applications, vol. 68, no. 6, pp. 1453-1461, 2008.

[2] B. Xiao and B. Liu, "Periodic solutions for Rayleigh type $p$-Laplacian equation with a deviating argument," Nonlinear Analysis: Real World Applications, vol. 10, no. 1, pp. 16-22, 2009.

[3] R. Manásevich and J. Mawhin, "Periodic solutions for nonlinear systems with $p$-Laplacian-like operators," Journal of Differential Equations, vol. 145, no. 2, pp. 367-393, 1998.

[4] W.-S. Cheung and J. Ren, "On the existence of periodic solutions for $p$-Laplacian generalized Liénard equation," Nonlinear Analysis: Theory, Methods E Applications, vol. 60, no. 1, pp. 65-75, 2005.

[5] W. Ge and J. Ren, "An extension of Mawhin's continuation theorem and its application to boundary value problems with a $p$-Laplacian," Nonlinear Analysis: Theory, Methods \& Applications, vol. 58, no. 3-4, pp. 477-488, 2004. 
[6] F. Zhang and Y. Li, "Existence and uniqueness of periodic solutions for a kind of Duffing type $p$ Laplacian equation," Nonlinear Analysis: Real World Applications, vol. 9, no. 3, pp. 985-989, 2008.

[7] B. Liu, "Existence and uniqueness of periodic solutions for a kind of Liénard type $p$-Laplacian equation," Nonlinear Analysis: Theory, Methods \& Applications, vol. 69, no. 2, pp. 724-729, 2008.

[8] W.-S. Cheung and J. Ren, "Periodic solutions for $p$-Laplacian Liénard equation with a deviating argument," Nonlinear Analysis: Theory, Methods \& Applications, vol. 59, no. 1-2, pp. 107-120, 2004.

[9] R. E. Gaines and J. L. Mawhin, Coincidence Degree, and Nonlinear Differential Equations, vol. 568 of Lecture Notes in Mathematics, Springer, Berlin, Germany, 1977.

[10] W.-S. Cheung and J. Ren, "Periodic solutions for $p$-Laplacian Rayleigh equations," Nonlinear Analysis: Theory, Methods E Applications, vol. 65, no. 10, pp. 2003-2012, 2006.

[11] S. Peng and S. Zhu, "Periodic solutions for $p$-Laplacian Rayleigh equations with a deviating argument," Nonlinear Analysis: Theory, Methods \& Applications, vol. 67, no. 1, pp. 138-146, 2007.

[12] F. Cao, Z. Han, and S. Sun, "Existence of periodic solutions for $p$-Laplacian equations on time scales," Advances in Difference Equations, vol. 2010, Article ID 584375, 13 pages, 2010.

[13] F. Cao and Z. Han, "Existence of periodic solutions for $p$-Laplacian differential equation with a deviating arguments," Journal of University of Jinan (Sci. Tech.), vol. 24, no. 1, pp. 95-98, 2010. 